\title{
Ultrafast laser ablation for targeted atherosclerotic plaque removal
}

\author{
Thomas Lanvin ${ }^{\mathrm{a}}$, Donald B. Conkey ${ }^{\mathrm{a}}$, Laurent Descloux ${ }^{\mathrm{a}}$, Aurelien Frobert ${ }^{\mathrm{b}}$, Jeremy Valentin ${ }^{\mathrm{b}}$, \\ Jean-Jacques Goy ${ }^{\mathrm{b}}$, Stéphane Cook ${ }^{\mathrm{b}}$, Marie-Noelle Giraud ${ }^{\mathrm{b}}$, and Demetri Psaltis*a \\ a Optics Laboratory, École Polytechnique Fédérale de Lausanne, 1015 Lausanne, Switzerland, \\ ${ }^{\mathrm{b}}$ Cardiology, Department of Medicine, University of Fribourg, 1700 Fribourg, Switzerland
}

\begin{abstract}
Coronary artery disease, the main cause of heart disease, develops as immune cells and lipids accumulate into plaques within the coronary arterial wall. As a plaque grows, the tissue layer (fibrous cap) separating it from the blood flow becomes thinner and increasingly susceptible to rupturing and causing a potentially lethal thrombosis. The stabilization and/or treatment of atherosclerotic plaque is required to prevent rupturing and remains an unsolved medical problem. Here we show for the first time targeted, subsurface ablation of atherosclerotic plaque using ultrafast laser pulses. Excised atherosclerotic mouse aortas were ablated with ultrafast near-infrared (NIR) laser pulses. The physical damage was characterized with histological sections of the ablated atherosclerotic arteries from six different mice. The ultrafast ablation system was integrated with optical coherence tomography (OCT) imaging for plaque-specific targeting and monitoring of the resulting ablation volume. We find that ultrafast ablation of plaque just below the surface is possible without causing damage to the fibrous cap, which indicates the potential use of ultrafast ablation for subsurface atherosclerotic plaque removal. We further demonstrate ex vivo subsurface ablation of a plaque volume through a catheter device with the high-energy ultrafast pulse delivered via hollow-core photonic crystal fiber.
\end{abstract}

Keywords: ablation, ultrafast laser, atherosclerosis, tissue, optical coherence tomography

\section{INTRODUCTION}

Heart disease is the most common cause of death worldwide, killing millions each year ${ }^{1}$. The main cause of heart disease, coronary artery disease, develops as immune cells and lipids accumulate into plaques within the wall of the coronary artery. As a plaque grows, the tissue layer (called the fibrous cap) separating it from the blood flow becomes thinner and increasingly susceptible to rupturing and causing a potentially lethal thrombosis ${ }^{2}$. When the fibrous cap becomes less than $65 \mu \mathrm{m}$ thick the plaque is considered unstable. The stabilization and/or treatment of unstable atherosclerotic plaque is required to prevent rupturing and remains a problematic medical condition out of the reach of existing plaque removal (atherectomy) devices. The laser atherectomy methods currently utilized are based on the ultraviolet excimer laser with nanosecond pulses. These lasers rely on linear absorption and thus are not fit for subsurface ablation ${ }^{3}$. Consequently, their use is limited to the extremities of the body where plaque rupture is less life threatening ${ }^{4}$ Here we demonstrate that ablation with ultrafast pulses (duration less than $10 \mathrm{ps}$ ) provides a mechanism to remove plaque without damaging the fibrous cap for prevention of plaque rupture during atherectomy. Subsurface ablation is enabled by the nonlinear absorption of the focused ultrafast pulse which confines the interaction to the focal volume $^{5}$. Ultrafast ablation occurs when laser irradiance surpasses an optical breakdown threshold over which multiphoton ionization leads to plasma formation ${ }^{6}$. A cavitation bubble forms with the expansion of material vaporized during this process. The result is a highly confined ablation region and a lingering volume of gas that eventually diffuses into the surrounding tissue ${ }^{7}$.

In this paper, we demonstrate targeted, ultrafast ablation of atherosclerotic plaques in free space and, for the first time, through a fiber-based catheter. We describe the results of an ex vivo investigation of the surface damage caused by ultrafast ablation in atherosclerotic plaque of a mice model. Furthermore, we monitor the ablation using optical coherence tomography (OCT), an imaging modality commonly used to characterize atherosclerotic plaques ${ }^{8}$. We find that ultrafast ablation of plaque just below the surface is possible without causing damage to the fibrous cap via our catheter prototype, which indicates the potential use of ultrafast ablation for subsurface atherosclerotic plaque removal. 


\section{EXPERIMENTAL METHODS}

The ablation experiments were performed on aortas extracted from genetically engineered mice lacking the apolipoprotein E (ApoE) gene'. The ApoE knock-out (ApoE-KO) mice developed atherosclerotic plaques within the tunica intima just a few microns below the endothelium of the arterial wall and grew to a couple hundred microns thick after being fed a lipid-rich diet. The plaques have a similar composition to human plaque with a fatty deposit and a thin fibrous cap ${ }^{10}$. Six animals were fed a lipid rich diet $(0.2 \%$ cholesterol and $21 \%$ butter, Western U8958 version 35 , SAFE, France) for 12 to 16 weeks. The mice were anesthetized and sacrificed prior to aorta harvesting. After harvesting the aortas were kept in phosphate buffer saline (PBS) solution on ice for 2 to 5 hours, afterwards the arteries were cut open along the longitudinal axis and mounted on a microscope slide in PBS solution with a cover slide. The tissues were then subjected to laser ablation with illumination from the endothelial side. All animals received humane care in compliance with the European Convention on Animal Care and in accordance with the Swiss Animal Protection Law after obtained permission of the State Veterinary Office, approved by the Swiss Federal Veterinary Office, Switzerland (FR 2013/35).
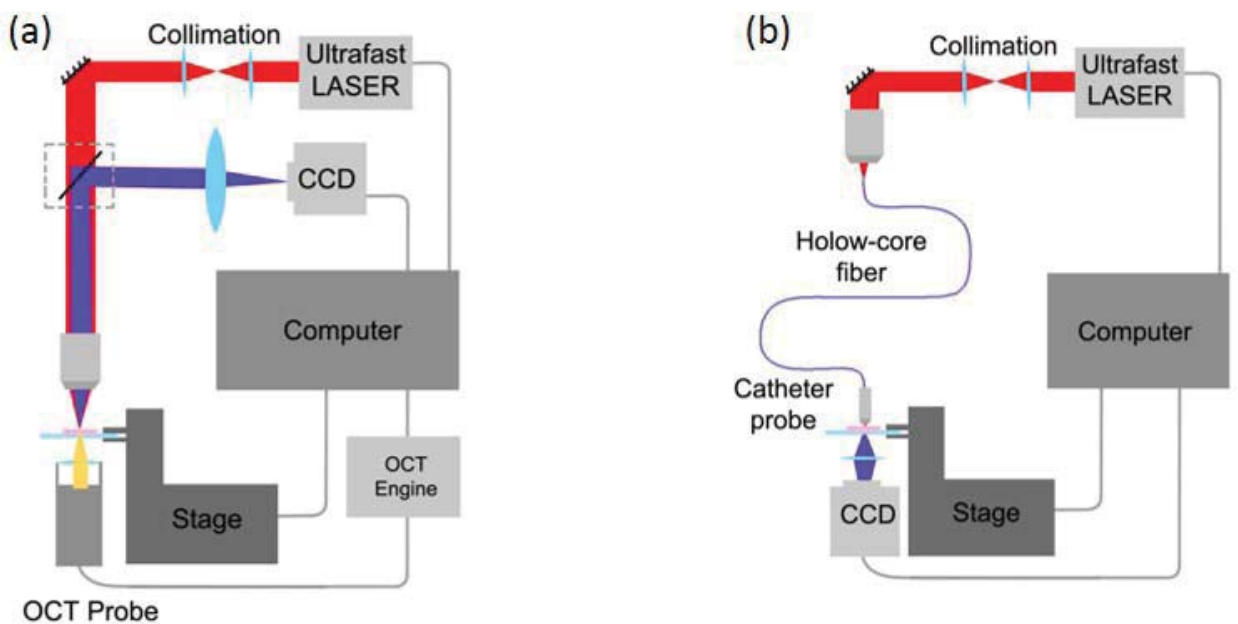

Figure 1. Schematics of the ablation setups. (a) The ablation setup for subsurface ablation analysis and OCT visualization. (b) The prototype catheter probe ablation setup.

Optical systems were built to facilitate the subsurface ultrafast ablation analysis (Figure 1(a)) and for initial ablation tests with a catheter probe prototype (Figure 1(b)). A beam from a 1030nm fiber laser (Satsuma HP2, Amplitude Systèmes) with tunable pulse width, repetition rate, and pulse energy provided high-energy, ultrafast laser input to the system. A 1.5 ps pulse duration, was selected to lessen nonlinear self-focusing effects ${ }^{11}$ while having ablation threshold values similar to femtosecond pulses ${ }^{12}$. With the repetition rate set at $1 \mathrm{kHz}$, pulse to pulse heat accumulation was avoided $^{13}$. For the subsurface ablation analysis the beam was collimated and expanded to fill the back aperture of a water immersion, long working distance objective (NA 0.8, providing FWHM focus spot of $0.8 \mu \mathrm{m}$ transverse and $1 \mu \mathrm{m}$ axial) mounted in an upright position. The objective focused the beam on the sample, which was mounted on a 3-axis piezo mechanical stage. To monitor the target area two imaging systems were integrated into the system: (1) a CCD camera provided a widefield image of the target area via a removable beamsplitter and (2) a spectral domain OCT system (Thorlabs, Ganymede) observed the same sample area from the posterior side. The OCT enabled depth-resolved visualization of the ablated tissue. For the prototype catheter setup (Figure 1(b)), the beam was coupled into an optical fiber for delivery to the catheter probe which is described in Section 5. The laser parameters, the stage motion, and both observation systems were controlled by computer. 
To facilitate subsurface ablation analysis, a specific ablation pattern was implemented by movement of the mechanical stage. The stage speed was adjusted to $v=(1-n) d f$, where $d$ is the FWHM of the focused laser spot, $f$ is the laser pulse repetition rate, and $n$ is the percentage overlap between subsequent pulses (set between 0 and 0.8 ). The pattern created either 8 or 10 ablation lines, with each line separated in the transverse (x) direction by $\delta_{\mathrm{x}}=30 \mu \mathrm{m}$ and in the axial (z) direction by $\delta_{\mathrm{z}}=10 \mu \mathrm{m}$ or $\delta_{\mathrm{z}}=15 \mu \mathrm{m}$. The number of lines and $\delta_{\mathrm{z}}$ were selected to allow for ablation through the entire artery.

To preserve the ablation holes for analysis, the ablated tissue samples were frozen and prepared for histologic sectioning. To ensure measurement of ablation hole size and not gas bubble size, a time greater than the observed gas bubble diffusion time ( $\sim 30 \mathrm{~min})$ elapsed following the ablation. The samples were then removed from the slide and immersed into cryogenic matrix (Cryomatrix, Thermo Scientific) and slowly frozen to $-20^{\circ} \mathrm{C}$ in the fumes of isopentane cooled by dry ice. Later, the samples were cut orthogonal to the ablation lines into $8 \mu \mathrm{m}$ sections using a cryocut and stained with haematoxylin and eosin $(\mathrm{H} \& \mathrm{E})$ for analysis of the ablated tissue.

\section{SUBSURFACE ABLATION ANALYSIS}

We analyzed the dependence of surface damage risk on pulse energy and ablation depth in plaque. The H\&E stained histological sections were evaluated under a transmission widefield microscope with a 40x objective (NA 0.7). The existence of surface damage was determined based on whether any structural modification existed above an ablation hole and near the surface of the tissue sample $(<10 \mu \mathrm{m})$. This damage was observed by visual inspection of the histological section as a structural deformation near the artery endothelium. For example, Figure 2(a) shows a row of ablation holes which move from deeper to shallower ablation depth from left to right. The first three holes from the left show no signs of surface damage, whereas the right-most hole shows a structural modification of the tissue near the surface. Figure 2(b) plots the percentage of ablation holes with surface damage as a function of pulse energy and depth of 1075 observed holes. As expected, the likelihood of surface damage decreases with increasing depth and decreasing energy. The results indicate that the risk of surface damage is minimal (around 5\%) when focusing deeper than $20 \mu \mathrm{m}$ under the surface with pulse energies below $2 \mu \mathrm{J}$. Importantly, this suggests the possibility of ablating vulnerable plaques without damaging thin fibrous caps.
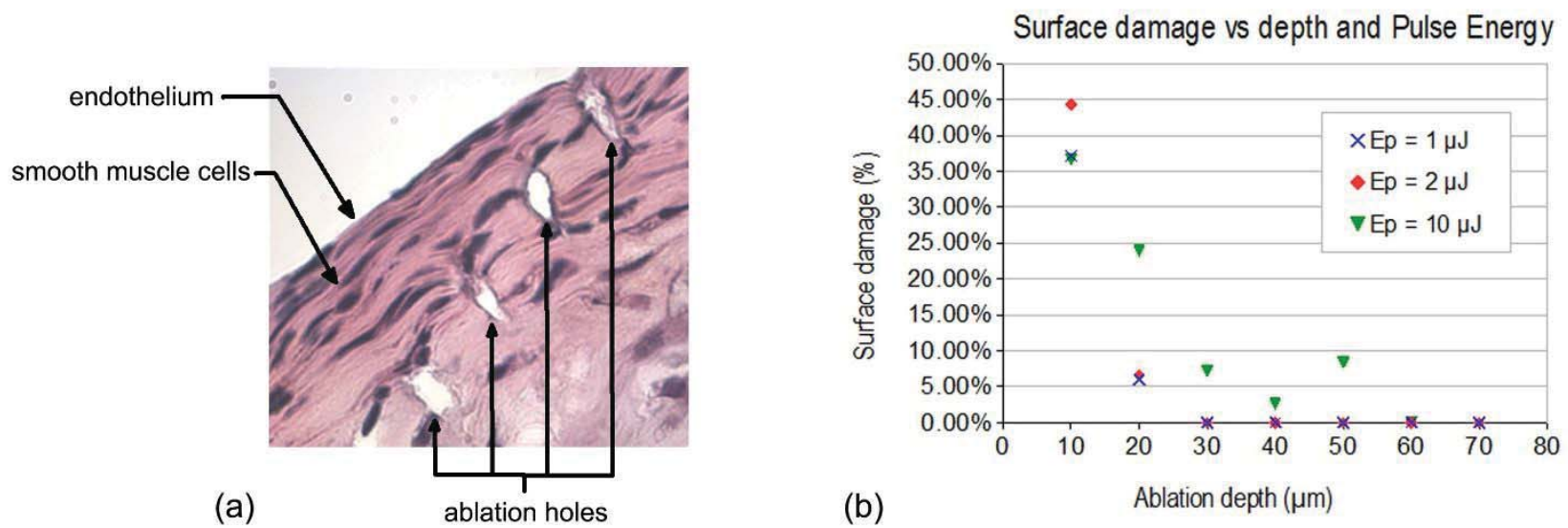

Figure 2. (a) A representative sample of ablated arterial tissue. (b) The percentage of ablation holes with surface damage as a function of pulse energy and depth.

\section{OCT OBSERVATION OF SUBSURFACE ABLATION}

The high refractive index difference between tissue and the vapor bubble resulting from ablation is well suited for OCT imaging. In the case of atherosclerotic plaque, OCT can be used to both locate the plaque and monitor the ablation. An initial test to image bulk subsurface ablation with OCT in a non-atherosclerotic mouse aorta (obtained from a C57/BL/6 mouse) was pursued. For bulk material ablation, a pattern was implemented to ablate a single plane of area $100 \times 200 \mu \mathrm{m}^{2}$ parallel to the tissue surface. The pattern was ablated with $1 \mu \mathrm{J}$ pulses targeting $50 \mu \mathrm{m}$ below the surface at 
two different locations. An OCT image acquired a few seconds after ablation showed two $30 \mu \mathrm{m}$ thick, gas filled volumes in the scanned area (Figure 3(a)). The sample was then frozen, sectioned, and stained with H\&E following the method described previously (Figure 3(b)). The ablation area appears clearly defined in the histological section without detectable structural damage near the surface. In general there is excellent agreement between the OCT and histology images where both show a pair of clearly defined ablation volumes.

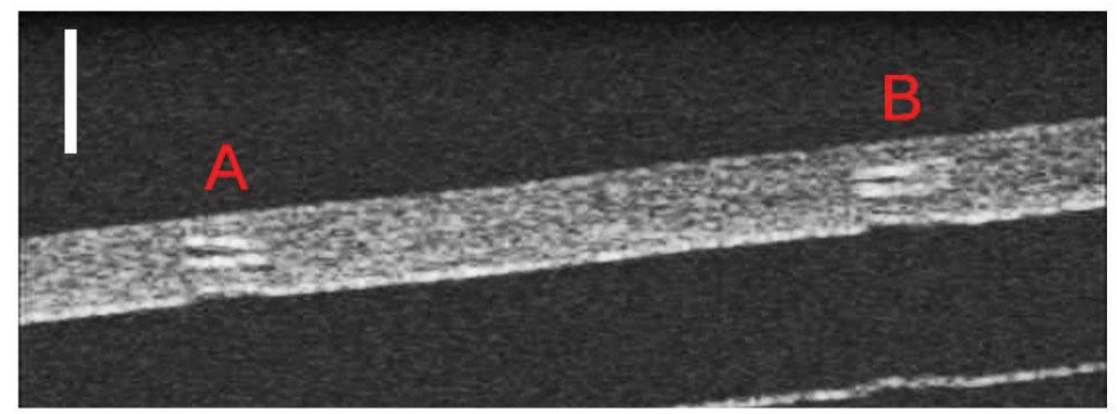

(a)

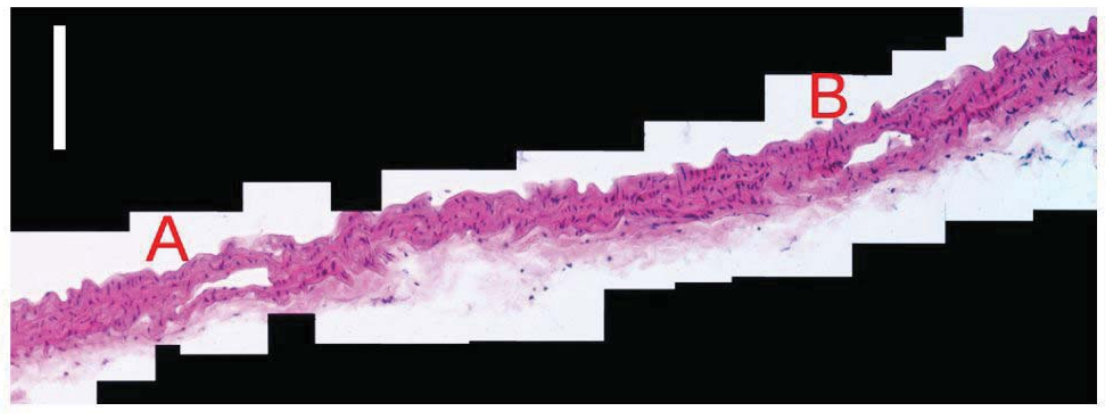

(b)

Figure 3. Tunica media of non-atherosclerotic mouse aorta ablated with a $100 \times 200 \mu \mathrm{m}^{2}$ planes. (a) Transverse OCT image of the ablation area. (b) Transverse histological cut of the ablation area. A and B mark the two different areas of ablation. The images are oriented in the same direction. The incident ablation pulse comes from the endothelium side. The scale bars are $100 \mu \mathrm{m}$.

\section{PROTOTYPE CATHETER PROBE}

The applicability of ultrafast ablation of atherosclerotic plaque depends on the ability to transfer the laser pulses through optical fibers to a catheter device. Fortunately, in the last decade there has been consistent progress in the maximum peak power which can propagate through single-mode hollow core photonic crystal fibers (HC-PCF) ${ }^{14}$. To demonstrate this capability, we built a prototype catheter probe to test subsurface ablation of plaque with light delivery through a HCPCF (Figure 4(a)). The $1.2 \mathrm{~mm}$ diameter probe houses the distal end of a single cell Kagome HC-PCF, for light delivery, with a lens (LightPath Technologies, 355070) to focus the light at a moderately high NA (0.6) into ApoE-KO plaque. For this initial demonstration the tissue sample was placed on the translation stage, while the catheter probe was stationary (Figure 1(b)). The tissue was scanned to target a $50 \mu \mathrm{m}$ deep, $70 \times 70 \mu \mathrm{m}^{2}$ plane. Following ablation the sample was prepared for histological sectioning following the method described previously. Figure 4(b) shows the H\&E stained slice of ablated tissue. The $70 \mu \mathrm{m}$ range of ablated plaque is visible below the red line. Further development of the catheter probe will enable in vivo testing of this method. 


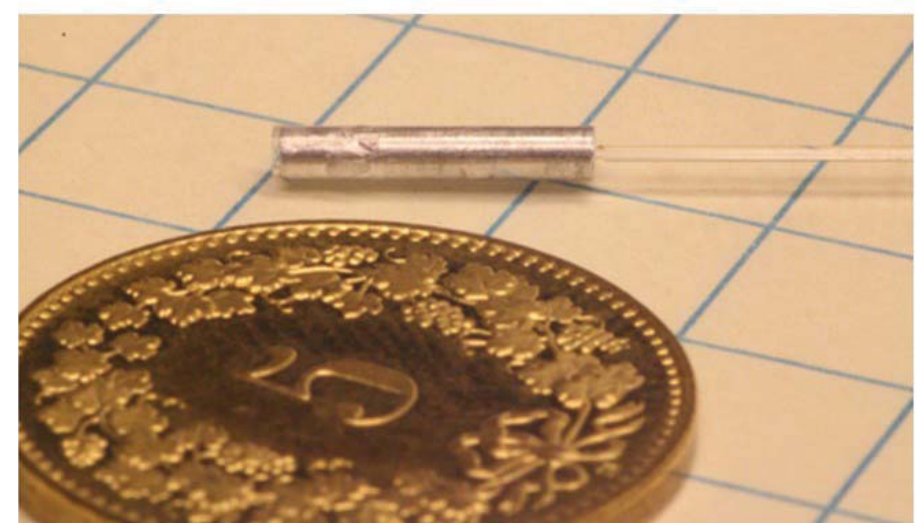

(a)

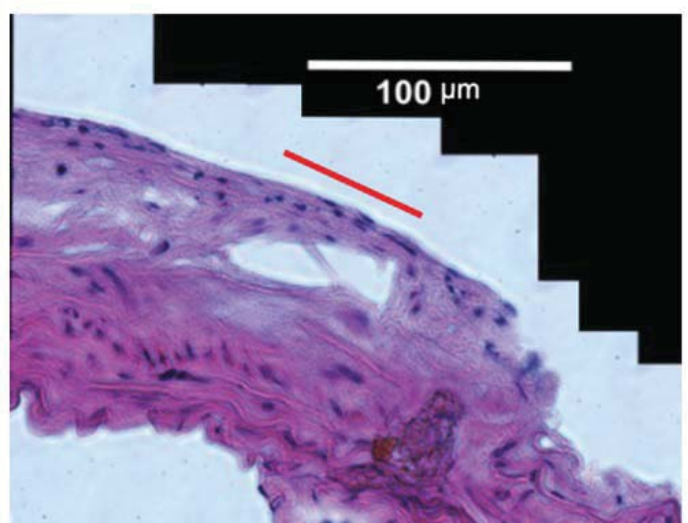

(b)

Figure 4. (a) Prototype catheter probe used to test ablation. (b) Subsurface ablation of plaque with the prototype catheter probe

\section{DISCUSSION}

We have analyzed subsurface ultrafast ablation in atherosclerotic plaque and tunica media tissue. We also demonstrated OCT imaging of the ablated volume which provided high contrast images of the gas bubble resulting from ablation. The use of OCT in conjunction with plaque ablation is convenient because IV-OCT devices already provide clinicians a high-resolution tool for plaque recognition and characterization ${ }^{15}$. The results showed that pulse energies below $4 \mu \mathrm{J}$ provided axially isolated ablation volumes, such that ablation targeted deeper than $20 \mu \mathrm{m}$ rarely caused surface damage. These results suggest the possibility of the fine ablation of unstable plaques only a few tens of microns beneath the endothelium. We have also presented the initial subsurface plaque ablation results from a catheter probe.

To better understand the effects of ultrafast ablation on plaque several experiments should be pursued. A study of the collateral cellular damage and reaction to ultrafast ablation would be informative because cell damage or death can extend beyond the ablation volume ${ }^{16}$. An experiment to examine the effects of ultrafast ablation on various plaque compositions should also be pursued. Also, investigating the long-term effects, such as inflammation and other immune responses, is necessary to understanding the medical implications of this approach. Of course, this study would require in vivo testing for which a further developed catheter prototype would be appropriate.

\section{CONCLUSION}

We have presented the initial steps towards the use of ultrafast ablation for atherosclerotic plaque treatment. We have characterized ultrafast ablation in atherosclerotic plaque performed on the atherosclerotic aortas of ApoE-KO mice. OCT provided high contrast images of the gas bubble resulting from ablation. The results indicate that pulse energies below 2 $\mu \mathrm{J}$ provide axially isolated ablation volumes, within the range of energy of our initial catheter prototype, such that ablation targeted deeper than $20 \mu \mathrm{m}$ rarely induce surface damage. These results suggest the possibility of the fine ablation of unstable plaques while preserving the stabilizing fibrous cap.

\section{ACKNOWLEDGMENTS}

We thank Thorlabs for lending the Ganymede OCT system. We acknowledge the support provided by the EPFL Histology Core Facility. D.B.C. acknowledges support from the 'EPFL Fellows' fellowship program co-funded by Marie Curie, FP7 Grant agreement no. 291771.

\section{REFERENCES}

[1] Finegold, J. A., Asaria, P.., Francis, D. P., "Mortality from ischaemic heart disease by country, region, and age: Statistics from World Health Organisation and United Nations," Int. J. Cardiol. 168(2), 934-945 (2013). 
[2] Fuster, V., Moreno, P. R., Fayad, Z. A., Corti, R.., Badimon, J. J., “Atherothrombosis and High-Risk Plaque: Part I: Evolving Concepts,” J. Am. Coll. Cardiol. 46(6), 937-954 (2005).

[3] Trokel, S. L., Srinivasan, R.., Braren, B., "Excimer Laser Surgery of the Cornea,” Am. J. Ophthalmol. 96(6), 710-715 (1983).

[4] Singleton, D. L., Paraskevopoulos, G., Taylor, R.., Higginson, L., "Excimer laser angioplasty: Tissue ablation, arterial response, and fiber optic delivery,” IEEE J. Quantum Electron. 23(10), 1772-1782 (1987).

[5] Du, D., Liu, X., Korn, G., Squier, J.., Mourou, G., "Laser-induced breakdown by impact ionization in SiO2 with pulse widths from 7 ns to 150 fs," Appl. Phys. Lett. 64(23), 3071-3073 (1994).

[6] Vogel, A., Busch, S.., Parlitz, U., "Shock wave emission and cavitation bubble generation by picosecond and nanosecond optical breakdown in water," J. Acoust. Soc. Am. 100(1), 148-165 (1996).

[7] Vogel, A., Noack, J., Hüttman, G.., Paltauf, G., "Mechanisms of femtosecond laser nanosurgery of cells and tissues," Appl. Phys. B 81(8), 1015-1047 (2005).

[8] Cilingiroglu, M., Oh, J. H., Sugunan, B., Kemp, N. J., Kim, J., Lee, S., Zaatari, H. N., Escobedo, D., Thomsen, S., et al., "Detection of vulnerable plaque in a murine model of atherosclerosis with optical coherence tomography," Catheter. Cardiovasc. Interv. 67(6), 915-923 (2006).

[9] Meir, K. S.., Leitersdorf, E., "Atherosclerosis in the Apolipoprotein E-Deficient Mouse A Decade of Progress," Arterioscler. Thromb. Vasc. Biol. 24(6), 1006-1014 (2004).

[10] Nakashima, Y., Plump, A. S., Raines, E. W., Breslow, J. L.., Ross, R., “ApoE-deficient mice develop lesions of all phases of atherosclerosis throughout the arterial tree," Arterioscler. Thromb. J. Vasc. Biol. Am. Heart Assoc. 14(1), 133-140 (1994).

[11] Hoy, C. L., Ferhanoglu, O., Yildirim, M., Kim, K. H., Karajanagi, S. S., Chan, K. M. C., Kobler, J. B., Zeitels, S. M.., Ben-Yakar, A., "Clinical Ultrafast Laser Surgery: Recent Advances and Future Directions," IEEE J. Sel. Top. Quantum Electron. 20(2), 242-255 (2014).

[12] Niemz, M. H., "Threshold dependence of laser-induced optical breakdown on pulse duration," Appl. Phys. Lett. 66(10), 1181-1183 (1995).

[13] Nguyen, J., Ferdman, J., Zhao, M., Huland, D., Saqqa, S., Ma, J., Nishimura, N., Schwartz, T. H.., Schaffer, C. B., "Sub-surface, micrometer-scale incisions produced in rodent cortex using tightly-focused femtosecond laser pulses," Lasers Surg. Med. 43(5), 382-391 (2011).

[14] Wang, Y., Alharbi, M., Bradley, T. D., Fourcade-Dutin, C., Debord, B., Beaudou, B., Gerôme, F.., Benabid, F., "Hollow-core photonic crystal fibre for high power laser beam delivery," High Power Laser Sci. Eng. 1(01), 1728 (2013)

[15] Suh, W. M., Seto, A. H., Margey, R. J. P., Cruz-Gonzalez, I.., Jang, I.-K., "Intravascular Detection of the Vulnerable Plaque," Circ. Cardiovasc. Imaging 4(2), 169-178 (2011).

[16] Qian, Z., Mordovanakis, A., Schoenly, J. E., Covarrubias, A., Feng, Y., Lilge, L.., Marjoribanks, R. S., "Pulsetrain-burst mode, ultrafast-laser interactions with 3D viable cell cultures as a model for soft biological tissues," Biomed. Opt. Express 5(1), 208-222 (2013). 